\title{
Percepciones de gestores de salud y facilitadores de la estrategia Atención Integrada a las Enfermedades Prevalentes (AIEPI) en una zona del Noreste de Brasil y Perú
}

\author{
Alfredo Borda-Olivas (a) \\ Matilde Palma-Ruiz ${ }^{(b)}$ \\ João Joaquim Freitas do Amaral(c)
}

Borda-Olivas A, Palma-Ruiz M, Amaral JJ. Perceptions of health managers and facilitators of the Integrated management of childhood illness (IMCI) in Northeastern Brazil and Peru Interface (Botucatu). 2017; 21(63):933-43.

The objective of this study was to explore the Integrated Management of Childhood Illness $(\mathrm{IMCl})$ strategy through the perceptions of the health managers and facilitators of strategy. A qualitative study was conducted using semi-structured interviews. Fourteen interviews were carried out, eight in the State of CearáBrazil and six in Peru, between May and June 2011. In general, participants expressed a positive perception of the $\mathrm{IMCl}$ strategy. Also was observed the predominance of the clinical component, compared with the community or the health services component. In Peru unlike Brazil, the government promoted the adoption of the strategy. In conclusion, this study shows a complex intervention of the $I \mathrm{MCl}$ strategy, with a predominance of the clinical component, evidencing the limitations in the integration of the components of the strategy.

Keywords: Integrated management of childhood illness. Qualitative research. Child health. Brazil. Peru.
El objetivo del estudio fue explorar las percepciones sobre la estrategia de Atención Integrada a las Enfermedades Prevalentes en la Infancia (AIEPI) de gestores de salud y facilitadores de la estrategia. Se realizó una investigación de tipo cualitativo mediante entrevistas semiestructuradas. Se llevaron a cabo 14 entrevistas entre mayo y junio del 2011, ocho en el Estado de Ceará-Brasil y seis en el Perú. En general los profesionales manifestaron una buena percepción de la estrategia AIEPI, observándose una predominancia del componente clínico, en comparación con el componente comunitario y de servicios de salud. A diferencia de Brasil, en el Perú hubo una promoción gubernamental para la adopción estrategia. En conclusión, este estudio muestra una intervención compleja de la estrategia AIEPI, con predominancia del componente clínico poniendo en evidencia las limitaciones en la integración de los componentes de la estrategia.

Palabras clave: Atención integrada a las enfermedades prevalentes en la infancia. Investigación cualitativa. Salud del niño. Brasil. Perú. (a,b) Agencia de Evaluación de Tecnologías Sanitarias, Instituto de Salud Carlos III. Calle de Sinesio Delgado, 4. Madrid, España.alfredoborda@ gmail.com;

mpalma@isciii.es

(c) Departmento de Saúde Materno Infantil Universidade Federal do Ceará. Fortaleza, CE, Brasil.joaoamaral@ terra.com.br 


\section{Introducción}

En 1996, la OPS/OMS y la UNICEF desarrollaron la estrategia Atención Integrada a las Enfermedades Prevalentes en la Infancia (AIEPI) con el objetivo de reducir la mortalidad infantil, la incidencia y gravedad de las enfermedades y los problemas de salud que afectan a los niños y niñas, principalmente infecciones respiratorias agudas (en particular neumonía), diarrea, malaria, desnutrición y sarampión, así como la mejora del crecimiento y desarrollo durante los cinco primeros años de vida ${ }^{1}$.

La estrategia AIEPI tiene como objetivo mejorar tres componentes principales: el desempeño de los profesionales de la salud; la organización del sistema de salud; y la práctica de la familia y la comunidad $^{2}$. El niño es tratado en base a intervenciones probadas que incluyen instrucciones paso a paso sobre la forma de evaluar a los niños y niñas enfermos de forma integral, las cuales son factibles de implementar en los países donde la mayoría de las muertes infantiles se producen ${ }^{3}$.

En el proceso de implementación de la estrategia se establecieron tres fases: la fase de introducción, cuyo propósito era asegurar la comprensión de la estrategia y sus implicancias por las autoridades de salud de los países beneficiarios; la fase de implementación temprana, con la puesta en marcha de la estrategia en un número limitado de distritos, para lo cual se requería la adaptación de las guías clínicas al entorno de un país específico, así como de políticas de salud; y por último, la fase de expansión para ampliar la gama de intervenciones de AIEPI a otros distritos del país ${ }^{4}$.

A lo largo de los años, la estrategia AEPI ha sido implementada en más de 113 países con ingresos bajos ${ }^{5}$ con una magnitud de aplicación variable. En el Brasil y Perú se implementaron entre los años 1996 y 1997, siendo en Brasil principalmente a nivel de los estados del Norte y Noroeste ${ }^{6}$, zonas del país con menores indicadores de salud; y en el Perú se implementó en diversas regiones del país con menores indicadores de salud, pero centralizada desde el Ministerio de Salud?

Las circunstancias del Brasil y Perú en los inicios de la estrategia AIEPI se caracterizaban por tener indicadores de salud con alta mortalidad y elevada prevalencia de enfermedades infectocontagiosas y desnutrición, siendo la tasa de mortalidad infantil (menores de 1 año) por mil nacidos vivos en

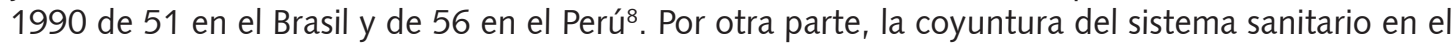
Brasil se desarrollaba en un contexto de fortalecimiento del sistema integrado de salud, a través de Sistema Único de Salud (SUS) ${ }^{9}$, el cual estaba organizado sobre la base de la descentralización al nivel municipal; en cambio en el Perú, el sistema de salud se caracterizaba por una fragmentación, con subsistemas brindando servicios de salud a diferentes segmentos de la población, con variados formas de financiamiento (Ministerio de Salud, Seguro Social en Salud-EsSalud, Fuerzas Armas y Policiales, y servicios privados) y un grupo grande, en su mayoría pobres, sin ningún tipo de cobertura de salud ${ }^{10}$.

A partir del año 1998 se empezó a realizar la evaluación multipaís de la estrategia AIEPI para medir su impacto en salud y la mortalidad de los niños, y evaluar si era costo-efectiva ${ }^{11}$. Perú y Brasil, además de Bangladesh, Tanzania y Uganda fueron los países seleccionados por algunas condiciones del país en relaciona a la estrategia como: la oportunidad en su implementación respecto a la evaluación, tamaño de la población cubierta por la estrategia, apoyo gubernamental y no gubernamental en la implementación de AIEPI y en las actividades de evaluación, estabilidad política de los países, presencia de áreas con diferencias en la prevalencia de malaria, entre otros ${ }^{12}$.

Los diversos estudios realizados en Perú y Brasil, y en otros entornos, han demostrado algunos beneficios y también limitaciones de la estrategia ${ }^{13}$; sin embargo, los estudios realizados no han abordado la evaluación de AIEPI considerando la integralidad de los tres componentes que lo constituyen, abordando los diversos puntos de vista, como puede ser desde la mirada de los gestores de la estrategia. 
En este sentido, este estudio tiene como objetivo explorar las percepciones de los gestores de salud y facilitadores de la estrategia AIEPI, con el fin de contar con información que contribuía a identificar debilidades y oportunidades de la estrategia, que pueda servir para plantear soluciones en otros ámbitos y países, a fin de reforzar las líneas estratégicas de promoción, prevención y acción de la salud infantil.

\section{Materiales y metodos}

En el presente estudio, de carácter exploratorio, se utilizó el diseño de investigación cualitativo, mediante entrevistas semiestructuradas, a personas claves, gestores de salud y facilitadores de la estrategia AIEPI, de organismos gubernamentales y no gubernamentales que tuvieron participación en algunas de las etapas de implementación de la estrategia AIEPI.

El ámbito de estudio en Brasil, debido a su extensión y por la forma de implementación de la estrategia principalmente en la zona del nordeste de Brasil ${ }^{14}$, se realizó en el Estado de Ceará, ubicada al nordeste del Brasil, cuya capital es Fortaleza, con una población estimada para el año 2010 de 8'448 055 habitantes, una cobertura del programa de Salud de la Familia (2009) del 71,9\% y una tasa de mortalidad infantil (2009) de 15,7 por mil nacidos vivos ${ }^{15}$. Por otro lado el Perú, con una población estimada para el año 2010 de 29'461933 habitantes, una población con protección en salud (2008) del $42 \%{ }^{10}$ y con una tasa de mortalidad infantil (2010) del 17 por mil nacidos vivos ${ }^{8}$.

Para las entrevistas se elaboró un guion semiestructurado, revisado y discutido (de forma presencial y virtual) entre los investigadores, a partir de la revisión de la literatura, sin descartar la posibilidad de que surgieran temas no contemplados en el mismo. El guion cubría los siguientes ejes temáticos: contexto epidemiológico y del sistema de salud; estructura, proceso y resultados de la estrategia AIEPI en sus tres componentes; puntos fuertes, necesidades sentidas y expectativas a futuro. Para la captación de los participantes se utilizó el muestreo no aleatorizado intencionado, seguido de muestreo en bola de nieve que continuó hasta la saturación de la información. En Ceará-Brasil (Fortaleza) se contactó a través de la Facultad de Medicina de la Universidad Federal de Ceará y en Perú (Lima) por intermedio de los entrevistados del Instituto Nacional de Salud del Niño.

El trabajo de campo se llevó a cabo entre mayo y junio del 2011. La duración de las entrevistas osciló entre 45 a 75 minutos. Uno de los investigadores (con lengua materna español y estudios completos de portugués, más una estancia de tres meses en Brasil) realizó las entrevistas en forma presencial en portugués (Fortaleza-Ceará), y en español (Lima-Perú). Se registraron en audio (el audio en portugués se tradujo al español) y transcribieron literalmente. Para el análisis, se realizó un análisis narrativo del contenido ${ }^{16}$, leyéndose repetidamente las transcripciones, para encontrar relaciones significativas entre los temas que surgían. Dicha información fue complementada con la revisión de la literatura, siendo finalmente los resultados consensuados entre los autores. El estudio fue revisado y aprobado en la Universidad Federal de Ceará y se solicitó el consentimiento informado verbal de los participantes antes de las entrevistas.

\section{Resultados}

Se realizaron catorce entrevistas a profesionales con experiencia en servicios de salud, 8 en CearáBrasil y 6 en el Perú. La mayoría de los entrevistados eran además profesores de universidades con actividades en servicios de salud infantil (Tabla 1). 
Tabla 1. Características de los entrevistados sobre la estrategia AIEPI en Ceará-Brasil y Perú. Año 2011.

\begin{tabular}{|c|c|c|c|c|}
\hline CODIGO & EDAD & PROFESION & LUGAR DE TRABAJO ACTUAL & EXPERIENCIA \\
\hline \multicolumn{5}{|c|}{ CEARÁ-BRASIL } \\
\hline $1 C$ & 45 & Médico-Pediatra & Funcionario de UNICEF & $\begin{array}{l}\text { Facilitadora de AIEPI en fase de } \\
\text { introducción e implementación. }\end{array}$ \\
\hline $2 C$ & 60 & Médico-Pediatra & $\begin{array}{l}\text { Docente de Medicina en Fortaleza, } \\
\text { Ceará. }\end{array}$ & $\begin{array}{l}\text { Facilitadora de AIEPI en fase de } \\
\text { introducción e implementación }\end{array}$ \\
\hline $3 C$ & $>60$ & Médico-Pediatra & $\begin{array}{l}\text { Docente de Medicina en Fortaleza, } \\
\text { Ceará. }\end{array}$ & $\begin{array}{l}\text { Facilitadora de AIEPI en fase de } \\
\text { introducción e implementación }\end{array}$ \\
\hline $4 C$ & $>60$ & Médico & $\begin{array}{l}\text { Promotor de la Fundación Oswaldo } \\
\text { Cruz en Ceará }\end{array}$ & $\begin{array}{l}\text { Promotor del Programa de Agentes } \\
\text { Comunitarios de Salud (PACS) desde } \\
\text { los } 80^{\prime} .\end{array}$ \\
\hline $5 C$ & $50-60$ & Enfermera & $\begin{array}{l}\text { Gestora de Municipio de Horizonte, } \\
\text { Ceará. }\end{array}$ & $\begin{array}{l}\text { Asesora y promotora del PACS desde } \\
\text { los años } 80^{\prime} .\end{array}$ \\
\hline $6 C$ & $50-60$ & Médico-Pediatra & $\begin{array}{l}\text { Docente de Medicina en Fortaleza, } \\
\text { Ceará. }\end{array}$ & $\begin{array}{l}\text { Facilitadora de AIEPI en fase de } \\
\text { introducción e implementación }\end{array}$ \\
\hline 7C & $50-60$ & Médico-Pediatra & $\begin{array}{l}\text { Personal del Hospital Estatal en } \\
\text { Fortaleza, Ceará. }\end{array}$ & $\begin{array}{l}\text { Facilitadora de AIEPI en fase de } \\
\text { introducción e implementación }\end{array}$ \\
\hline $8 C$ & $50-60$ & Neonatología & $\begin{array}{l}\text { Colaborador en la secretaria de salud } \\
\text { pública de Ceará. }\end{array}$ & Atención de salud pediátrica-neonatal \\
\hline \multicolumn{5}{|c|}{ PERU } \\
\hline $1 \mathrm{P}$ & $>60$ & Médico-Pediatra & $\begin{array}{l}\text { Docente, funcionaria del Instituto } \\
\text { Nacional de salud del Niño. }\end{array}$ & Colaborador de AIEPI. \\
\hline $2 \mathrm{P}$ & $50-60$ & Médico-Pediatra & $\begin{array}{l}\text { Docente y personal de Instituto } \\
\text { Nacional de salud del Niño. }\end{array}$ & $\begin{array}{l}\text { Facilitador de AIEPI en fase de } \\
\text { introducción e implementación. }\end{array}$ \\
\hline $3 P$ & $50-60$ & Médico-Pediatra & Funcionario de la OPS & $\begin{array}{l}\text { Promotor de AIEPI en el Perú, como } \\
\text { funcionario del MINSA y OPS. }\end{array}$ \\
\hline $4 \mathrm{P}$ & $50-60$ & $\begin{array}{l}\text { Médico, Máster de } \\
\text { Salud Pública }\end{array}$ & $\begin{array}{l}\text { Funcionaria del Ministerio de Salud } \\
\text { en el área de atención infantil. }\end{array}$ & Colaboradora de AIEPI. \\
\hline $5 P$ & $30-40$ & Médico & $\begin{array}{l}\text { Jefe de establecimiento de salud de } \\
\text { nivel primario de Ayacucho. }\end{array}$ & $\begin{array}{l}10 \text { años en atención primaria de la } \\
\text { salud. }\end{array}$ \\
\hline $6 P$ & $30-40$ & Médico & $\begin{array}{l}\text { Jefe de establecimiento de salud de } \\
\text { nivel San Martín }\end{array}$ & $\begin{array}{l}10 \text { años en atención primaria de la } \\
\text { salud. }\end{array}$ \\
\hline
\end{tabular}

El análisis de las entrevistas y la comparación de los discursos de los profesionales sobre la estrategia AIEPI permitieron identificar diversos contenidos organizados en las siguientes áreas temáticas, que se ejemplifican (entre comillas) con fragmentos de las narraciones de las personas participantes.

\section{Visión general de la estrategia AIEPI}

En general los profesionales manifestaron una buena percepción de la estrategia AIEPI, aunque señalaron dificultades para su evaluación en términos de indicadores de salud y mortalidad infantil: "En la medida que veamos (solo) los indicadores, no va a ser fácil decir que (mejoras) se deben solo a la estrategia AIEPI" (2P).

En el Perú, los informantes manifiestan una percepción positiva del impacto: "(La estrategia) AIEPI es una excelente estrategia [...] debe introducirse en el comportamiento del médico desde que el médico se está formando, al igual que a las enfermeras, las asistentas sociales, o sea todo el equipo de salud" (1P).

Pero también manifiestan diversos problemas; por ejemplo, relacionados a los procesos administrativos, puntualmente con la gestión de los recursos económicos a través del mecanismo de presupuesto por resultado, los cuales según señalan pueden generar procesos desintegradores: "Incluso 
ahora que se habla de presupuesto por resultado, se realiza (atenciones de) IRA (Infección Respiratoria Aguda), atenciones de EDA (Enfermedad Diarreica Aguda), (atenciones de) inmunizaciones, cada uno con su propio presupuesto por resultados" (1P).

En el Brasil, los informantes admiten cierta competencia de la estrategia AIEPI con otros programas sociales: "Aquí la influencia (de la estrategia AIEPI) fue pequeña, porque fue muy poco utilizada [...] el programa bolsa de familia y (el programa de) jubilación de ancianos [...], eso sí realmente generaron grandes cambios" (4C).

\section{Contexto del Sistema de Salud y epidemiológico}

En Brasil a finales de la década de los 80' se creó el Sistema Único de Salud, con la municipalización de la salud y el Programa de Agentes Comunitarios de Salud (PACS). Posteriormente se creó el Programa de Salud de la Familia- (PSF), estrategia principal de estructuración de la atención básica de los sistemas locales de salud.

“La creación del SUS coincide con el movimiento de la redemocratización del país [...] la introducción del agente comunitario de salud se da en momentos que se implementa la descentralización de la salud". (5C)

En el Perú, a partir de la década del 90', hubo un cambio en el nivel político, económico y social. Se creó el Seguro de Escolar gratuito en 1997, posteriormente ampliado al actual Seguro Integral de Salud (SIS). Los entrevistados perciben el Sistema de Salud como deficiente y limitado en recursos humanos y materiales: "Los establecimientos de salud de atención primaria están muy descuidados, muy poco abastecidos, (el personal) muy poco capacitados" (1P).

Respecto al contexto epidemiológico, en los inicios de la estrategia AIEPI, en ambos países se observaba una alta mortalidad y prevalencia elevada de enfermedades infecto-contagiosas y desnutrición.

\section{Desarrollo de la estrategia AIEPI}

Los profesionales de ambos países destacaron la participación de las diferentes instituciones, como las universidades, el ministerio de salud y sociedades de salud, bajo la dirección de la OPS. En el proceso de introducción se realizó la traducción y adaptación de los materiales de trabajo al entorno específico, con algunos matices.

En el Perú hubo una adopción gubernamental de la estrategia como parte del Modelo de Atención Infantil de la Salud (MAIS):

\footnotetext{
“Hay una resolución que adopta la estrategia AIEPI [...]. El Ministerio de Salud lo introduce como estrategia válida para la implementación de la atención integral en la etapa de vida niño [...] hemos adaptado la historia (clínica), la hemos convertido en una ficha única, basada en las priorizaciones, en la evaluación integral del niño". (3P)
}

En cambio en el Brasil, los profesionales señalaron que no se incluyó la sistemática de la estrategia AIEPI en la atención de salud infantil, mencionan además que el gobierno de Brasil, redujo su participación en la capacitación de la estrategia AIEPI a pesar de su importancia para la salud infantil: "La estrategia AIEPI no está afianzada ni a nivel del país, ni del Estado [...]. Se suspendió el apoyo al AIEPI en los últimos 8 años" (2C).

\footnotetext{
"La viabilidad técnica de (la estrategia) AIEPI es innegable, todos concuerdan de que es un buen programa. La viabilidad financiera también, las capacitaciones no son caras [...]. En este momento lo que falta es la viabilidad política". (6C)
} 
Dicha información es refrendada en una publicación donde señalan que el gobierno del Brasil, desde el año 2003 redujo su participación en la capacitación en AIEPI, redefiniendo sus prioridades en la atención de salud infantil ${ }^{17}$.

\section{El componente clínico de la estrategia AIEPI}

El componente clínico es el componente que según los participantes, tuvo un mayor desarrollo en ambos país. En el Brasil, las personas entrevistadas informaron que se realizó una capacitación masiva inicial a las enfermeras y médicos generales, aunque con poco seguimiento. No hubo una priorización específica y se observó una alta rotación del personal: "En su inicio se realizó mucha capacitación. Primero para los médicos, luego para los enfermeros [...]. Hubo un momento que todo el mundo quería hacer AIEPI [...]; (pero) no hubo seguimiento [...] se realizaba según el interés del municipio" (6C).

En el Perú, según las personas participantes, se llevó a cabo una adecuación de la capacitación tanto en duración (modificando de dos semanas a una) como en la modalidad. Inicialmente la capacitación fue presencial y luego se impulsó la formación a distancia: "El curso clínico de (la estrategia) AIEPI está totalmente virtualizado para la capacitación a distancia. La OMS logró desarrollar un software, el ICATT (IMCI Computerized Adaptation and Training Tool, Material Computarizado para la Adaptación y Capacitación de AIEPI)" (3P).

También se emprendió una selección de regiones prioritarias de acuerdo a las sus condiciones de salud, pero refieren una alta rotación del personal sanitario al cabo de uno o dos años.

\section{El componente comunitario de la estrategia AIEPI}

Los participantes señalaron, en ambos países, la escasa implementación de este componente. En Brasil, manifiestan una poca implementación, a pesar de tener el PACS, en la que la figura del agente comunitario está integrado al SUS (con remuneración), realizando actividades en la familia y la comunidad paralelas a la estrategia AIEPI:

\footnotetext{
“(La estrategia) AIEPI no fue una prioridad (orientada a) los agentes comunitarios [...], el componente más enfatizado en Brasil fue en la capacitación de los profesionales [...]. Hubo fallas respecto a la organización de los servicios y sobre todo en la comunidad [...], a pesar de tener un gran programa de agentes comunitarios de salud". (3C)
}

En el Perú, también manifiestan un predominio en la implementación del componente clínico, pero refieren un interés de involucrar agentes comunitarios (voluntarios) y a otros líderes sociales (alcaldes, profesores): "Las herramientas que más nos han ayudado es del (componente) clínico [...]. Se escribió el material de las prácticas dirigidas al alcalde [...] a los maestros" (3P).

\section{El componente de los servicios de salud de la estrategia AIEPI}

En ambos países los entrevistados evidencian una percepción poco definida respecto a este componente. En Brasil, el entorno del sistema de salud es más favorable, con un sistema descentralizado y programas de salud integrados.

En el Perú, el entorno parece menos propicio, por la falta de organización del sistema de salud y fragmentación de los niveles de atención de salud: " [...] hay una suerte de perversión del sistema, son tres niveles de atención pero las relaciones no son de abajo arriba, si no que se saltan" (1P). 


\section{Discusion}

En el presente estudio, uno de los resultados más importantes está relacionado con el proceso de la implementación de la estrategia AIEPI, donde se observa una intervención muy compleja, en varias fases y componentes, con una predominancia del componente clínico en comparación con los otros dos componentes de la estrategia. Ello podría estar evidenciando limitaciones de la estrategia en cuanto al desarrollo coordinado e integrado de los componentes que constituyen la estrategia. Por otro lado, se observa que en el Perú el gobierno promovió y oficializó (mediante resolución ministerial), la incorporación de AIEPI como estrategia de intervención dentro del Modelo de Atención Integral de Salud ${ }^{18}$; en cambio en Brasil, aunque en las etapas iniciales se observa un impulso del componente de capacitación de los profesionales ${ }^{19}$, en los años posteriores los programas e iniciativas desarrolladas en salud infantil ${ }^{20}$, aunque pudieran haber recogido conceptos o actividades de la estrategia, éstas no fueran explicitadas o se desarrollaron al margen de la estrategia AIEPI.

Los resultados señalados en el presente estudio confirman los hallazgos de otras publicaciones. Así, Ahmend et al ${ }^{13}$ remarcan que en muchos países sólo se ha puesto en práctica el componente de capacitación del personal sin una reforma complementaria de los sistemas o la política de salud. Del mismo modo, Chopra $M$. et al ${ }^{21}$ en una publicación, señala que en la mayoría de los países, el componente comunitario y de los servicios de salud nunca fueron implementados o fueron implementados de manera limitada o inadecuada, observándose limitaciones en los sistemas de referencias y supervisión, y falta de suministros de medicamentos.

Una aproximación que pueden explicar estos hallazgos del estudio, pueden estar relacionados a la priorización desde el momento de la concepción de la estrategia del componente clínico, en razón de la identificación a priori, de las condiciones de debilidad y fragmentación de los sistemas de $\operatorname{salud}^{22}$ y de las deficiencias en la estructura y organización de los actores de salud del componente comunitario y de la familiar ${ }^{23}$. Asimismo, respecto al componente de la práctica de la familia y la comunidad, la consideración del agente comunitario como un recurso humano en salud complementario ante la escasez de trabajadores de la salud ${ }^{24}$, y no como un vínculo entre la población y los servicios de salud, podría haber condicionado un poco empoderamiento de los agentes comunitarios, y por ende un limitado desarrollo del componente comunitario de la estrategia.

Por otro lado, la participación limitada de los gobiernos en la implementación de la estrategia AIEP ${ }^{17}$; con un menor involucramiento de autoridades locales con la necesaria autoridad y jerarquía, y dificultades en la coordinación con los organismos impulsores de la estrategia (ONU, OMS, UNICEF) ${ }^{25}$, podría explicarse por la insuficiente participación de los países beneficiarios en la concepción de la estrategia ${ }^{1}$, lo que podría haber condicionado un menor involucramiento en la implementación y expansión de la estrategia. Distinto a ello se observa en Brasil, con el desarrollo de diversas iniciativas concebidas dentro del ámbito local que surgieron y se desarrollaron en los periodos de la estrategia AIEPI como: la creación de comités para la prevención de la mortalidad infantil (2005), el pacto por la vida, para la reducción de la mortalidad (2006), iniciativas específicas (promoción de la inmunización, la lactancia materna y el alojamiento conjunto), mejoras en el acceso a la atención preventiva y curativa de la salud, incluyendo el SUS, el PSF, el PACS, de la Pastoral y, otros políticas y programas sociales que repercutieron en la mejora de la salud infanti ${ }^{20,26}$.

No obstante a lo señalado previamente, es necesario resaltar que, frente a nuestros hallazgos que podrían considerarse poco alentadores, por otros aspectos de la estrategia, principalmente los relacionados del componente clínico, en estudios realizados en el Perú 7 14,27, Brasi ${ }^{14,28,29}$ y en otros países del entorno, se observa diversos beneficios de la estrategia AIEPI; por ejemplo, en la formación y rendimiento de los profesionales en la atención de salud infantil ${ }^{6,30}$, reducción de los costos globales de los servicios de salud ${ }^{31}$ mejoras en el uso racional de medicamentos ${ }^{32}$, entre otros. Asimismo, un aporte importante de la estrategia ha sido el reforzamiento del concepto de la atención de salud integral, frente a la atención de salud fragmentada en programas verticales ${ }^{33}$, sin tener en cuenta la frecuente superposición de síntomas y la posible presencia de más de una enfermedad. Dichas concepción de atención, en los momentos actuales se hace necesario relanzar, frente a los nuevos modelos y perspectivas de atención de salud, y de políticas de salud, orientadas a la privatización y a 
la promoción de asociaciones público privadas de la salud ${ }^{34,35}$, en los cuales en algunos de los casos podrían orientarse a un afán puramente económico, que podría colisionar con la necesidad de una atención integral de la salud, no solo en el campo de la atención infantil, sino también en la atención de salud en cada una de las etapas de vida ${ }^{36}$.

Los hallazgos presentados en este estudio deben ser interpretados tomando en cuenta algunas limitaciones del estudio. En primer lugar, la posibilidad de sesgo de información debido a la vinculación profesional de los entrevistados e investigadores, lo cual se procuró controlar, mediante el muestro en bola de nieve, solicitándose a los entrevistados identificar otras personas que puedan incluirse como entrevistados; sin embargo, consideramos que si existió alguna vinculación entre entrevistados e investigadores esta podría haber facilitado un mejor recojo de la información. En segundo lugar, la mayoría de las personas entrevistadas pertenecía a similar contexto académico, ello se explica por la orientación de la estrategia AIEPI, es decir, hacia los profesionales encargados de la atención infantil. Por último, se realizó poca profundización respecto a las percepciones del componente comunitario y de servicios de salud, lo cual se debió a la dificultad de identificar informantes claves con experiencia en dichos temas, que podría explicarse por el limitado desarrollo de estos componentes. Un desarrollo de entrevistas a profesionales asistenciales y agentes comunitarios podrían haber enriquecido el análisis, cuestiones que podrían ser abordadas en un nuevo estudio.

En conclusión, en esta investigación se observa una intervención compleja de la estrategia AIEPI, con predominancia del componente clínico respecto al componente comunitario y de servicios de salud, poniendo en evidencia las limitaciones en la integración de los componentes de la estrategia AIEPI, lo que hace necesario un replanteamiento de la misma, con una orientación a su alcance real, es decir, al componente clínico, con mayores esfuerzos en la formación del personal de salud, involucrando también al sector privado, a fin de reforzar las líneas estratégicas de promoción, prevención y acción de la salud infantil.

\section{Colaboradores}

Alfredo Borda-Olivas ha participado en la concepción del artículo, la recolección y análisis de datos, redacción y aprobación de la versión final. Matilde Palma-Ruiz ha participado en la concepción del artículo, el análisis de datos, corrección de los borradores y aprobación de la versión final. Joao Amaral ha participado en la recolección de datos, corrección de los borradores y aprobación de la versión final. 


\section{Referências}

1. Gove S. Integrated management of childhood illness by outpatient health workers: technical basis and overview. The WHO Working Group on Guidelines for Integrated Management of the Sick Child. BullWorld Health Organ. 1997; 75Suppl 1:7-24.

2. Tulloch J. Integrated approach to child health in developing countries. Lancet. 1999; 354Suppl 2:SII16-20.

3. Jones G, Steketee RW, Black RE, Bhutta ZA, Morris SS. How many child deaths can we prevent this year? Lancet. 2003; 362(9377):65-71.

4. World Health Organization, UNICEF. IMCI information package. 1999 [citado 27 Mar 2014]. Disponible: http://www.who.int/maternal_child_adolescent/documents/ chs_cah_98_1a/en/.

5. Lange S, Mwisongo A, Mæstad O. Why don't clinicians adhere more consistently to guidelines for the Integrated Management of Childhood Illness (IMCI)? Soc Sci Med. 2014; 104:56-63.

6. Amaral JJ, Victora CG, Leite AJ, Cunha AJ. Implementation of the Integrated Management of Childhood Illnesses strategy in Northeastern Brazil. Rev Saude Publica. 2008; 42(4):598-606.

7. Pereyra Zaldívar H. Los diez primeros años de AIEPI en el Perú. Lima: Organización Panamericana de la Salud; 2006.

8. World Health Organization. Global Health Observatory Data Repository [Internet]. Geneva: WHO; 2011 [citado 17 Jun 2016]. Disponible: http://apps.who.int/gho/data/ node.home.

9. Paim J, Travassos C, Almeida C, Bahia L, Macinko J. The Brazilian health system: history, advances, and challenges. Lancet. 2011; 377(9779):1778-97.

10. Alcalde-Rabanal JE, Lazo-González O, Nigenda G. The health system of Peru. Salud Publica Mexico. 2011; 53Suppl 2:s243-54.

11. Huicho L, Davila M, Campos M, Drasbek C, Bryce J, Victora CG. Scaling up integrated management of childhood illness to the national level: achievements and challenges in Peru. Health Policy Plan. 2005; 20(1):14-24.

12. Bryce J, Victora CG, Habicht JP, Vaughan JP, Black RE. The multi-country evaluation of the integrated management of childhood illness strategy: lessons for the evaluation of public health interventions. Am J Public Health. 2004; 94(3):406-15.

13. Ahmed HM, Mitchell M, Hedt B. National implementation of integrated management of childhood illness (IMCI): policy constraints and strategies. Health Policy. 2010; 96(2):128-33.

14. Benguigui Y, Bossio JC, Arias SJ. AIEPI en las Américas: enfermedades prevalentes de la infancia en las Américas. Washington: Organización Panamericana de la Salud; 2008.

15. Governo do Estado do Ceará. Ceará em números [Internet] [citado 28 Mar 2014]. Disponible: http://www.ceara.gov.br/ceara-em-numeros.

16. Amezcua M, Gálvez Toro A. Modes of analysis in qualitative research in health: critical perspective and spoken reflexions. Rev Esp Salud Publica. 2002; 76(5):423-36.

17. Amorim DG, Adam T, Amaral J, Gouws E, Bryce J, Victora CG. Integrated management of childhood illness: efficiency of primary health in Northeast Brazil. Rev Saude Publica. 2008; 42(2):183-90.

18. Ministerio de Salud del Perú. Resolución Ministerial No 506-2005/MINSA que oficializa la "Atención Integrada de las Enfermedades Prevalentes de la Infancia" (AIEPI) como estrategia de intervención en el Modelo de Atención Integral de Salud. Diario Oficial El Peruano. Jul 2005; 296150-1. 
19. Ministerio de Salud de Brasil. AIDPI Atenção Integrada às Doenças Prevalentes na Infância: curso de capacitação: introdução: módulo 1 Ministério da Saúde, Organização Mundial da Saúde, Organização Pan-Americana da Saúde. 2a ed. Brasília: Ministério da Saúde; 2002. Disponible: http://bvsms.saude.gov.br/bvs/publicacoes/AIDPI_modulo_1. pdf.

20. Victora CG, Aquino EML, do Carmo Leal M, Monteiro CA, Barros FC, Szwarcwald CL. Maternal and child health in Brazil: progress and challenges. Lancet. 2011; 377(9780):1863-76.

21. Chopra M, Binkin NJ, Mason E, Wolfheim C. Integrated management of childhood illness: what have we learned and how can it be improved? Arch Dis Child. 2012; 97(4):350-4.

22. World Health Organization. The analytic review of the Integrated Management of Childhood Illness strategy [Internet]. 2003 [citado 27 Mar 2014]. Disponible: http://www. who.int/maternal_child_adolescent/documents/9241591730/en/.

23. Borda-Olivas A, Palma-Ruiz M, Drasbek C, Sarria-Santamera A. Community health workers: a literature review in some countries of South America. Value Health. 2012; 15(4):A27-8.

24. World Health Organization. The world health report: 2006: working together for health [Internet]. Genebra: WHO; 2006 [citado 18 Nov 2014]. Disponible: http://apps. who.int/iris/handle/10665/43432.

25. Victora CG, Adam T, Bryce J, Evans DB. Integrated management of the sick child. In: Jamison DT, Breman JG, Measham AR, Alleyne G, Claeson M, Evans DB, et al., editors. Disease control priorities in developing countries [Internet]. Washington (DC): World Bank; 2006 [citado 27 Mar 2014]. Disponible: http://www.ncbi.nlm.nih.gov/books/ NBK11752/.

26. Frias PG, Mullachery PH, Giugliani ERJ, Frias PG, Mullachery PH, Giugliani ERJ. Políticas de saúde direcionadas às crianças brasileiras: breve histórico com enfoque na oferta de serviços de saúde. In: Ministério da Saúde, editor Saúde Brasil 2008: 20 anos do Sistema Único de Saúde no Brasil [Internet]. Brasília: Ministério da Saúde; 2009 [citado 15 Jul 2016]. p. 85-110. Disponible: http://bvsms.saude.gov.br/bvs/publicacoes/saude_ brasil_2008.pdf.

27. Dávila $M$, Pereyra $H$. Investigaciones relacionadas con el componente comunitario de AIEPI. Washington: Organización Panamericana de la Salud; 2003.

28. Amaral J. Manejo de casos da AIDPI nas unidades de saúde no Brasil-2002. Unpublished report; 2002.

29. Cunha ALA, Silva MAF, Amaral J. A estratégia de "Atenção Integrada às Doenças Prevalentes na Infância-AIDPI" e sua implantação no Brasil. Rev Pediatr Ceara. 2001; 2(1):33-8.

30. Harkins T, Drasbek C, Arroyo J, McQuestion M. The health benefits of social mobilization: experiences with community-based Integrated Management of Childhood Illness in Chao, Peru and San Luis, Honduras. Promot Educ. 2008; 15(2):15-20.

31. Adam T, Edwards SJ, Amorim DG, Amaral J, Victora CG, Evans DB. Cost implications of improving the quality of child care using integrated clinical algorithms: evidence from Northeast Brazil. Health Policy. 2009; 89(1):97-106.

32. Gouws E, Bryce J, Habicht J-P, Amaral J, Pariyo G, Schellenberg JA, et al. Improving antimicrobial use among health workers in first-level facilities: results from the multicountry evaluation of the Integrated Management of Childhood Illness strategy. Bull World Health Organ. 2004; 82(7):509-15. 
33. Claeson M, Waldman RJ. The evolution of child health programmes in developing countries: from targeting diseases to targeting people. Bull World Health Organ. 2000; 78(10):1234-45.

34. La Forgia GM, Harding A. Public-private partnerships and public hospital performance in São Paulo, Brazil. Health Aff. 2009; 28(4):1114-26.

35. Zevallos L, Salas V, Robles L. Public-private partnerships for health services: the solution for the peruvian health system? Rev Peru Med Exp Salud Publica. 2014; 31(4):769-74.

36. Salinsky E. Medicine, big business, and public health: wake up and smell the starbucks. Prev Chronic Dis [Internet]. 2009 [citado 19 Nov 2015]; 6(2):A75. Disponible: http://www.ncbi.nlm.nih.gov/pmc/articles/PMC2687881/.

Borda-Olivas A, Palma-Ruiz M, Amaral JJ. Percepções de gestores de saúde e facilitadores da estratégia Atenção Integrada às Doenças Prevalentes na Infância (AIDPI) em uma área do Nordeste do Brasil e Peru. Interface (Botucatu). 2017; 21(63):933-43.

O objetivo do estudo foi explorar as percepções da estratégia de Atenção Integrada às Doenças Prevalentes na Infância (AIDPI) de gestores de saúde e facilitadores da estratégia. A pesquisa qualitativa foi realizada por meio de entrevistas semiestruturadas. Foram realizadas 14 entrevistas, entre maio e junho de 2011, oito no Estado do Ceará-Brasil e seis no Peru. De um modo geral, os profissionais relataram uma boa percepção da estratégia AIDPI. Observou-se a predominância do componente clínico, em comparação com os serviços de componentes da comunidade e de saúde. Ao contrário do Brasil, no Peru, houve uma promoção do governo para adoção da estratégia. Em conclusão, este estudo mostra uma intervenção complexa da estratégia AIDPI, com predomínio do componente clínico, colocando em evidência as limitações na integração dos componentes da estratégia.

Palavras-chave: Atenção integrada às doenças prevalentes na infância. Pesquisa qualitativa. Saúde da criança. Brasil. Peru. 
